\title{
Alternative epithelial markers in sarcomatoid carcinomas of the head and neck, lung, and bladder-p63, MOC-31, and TTF-1
}

\author{
James S Lewis Jr, Jon H Ritter and Samir El-Mofty \\ Department of Pathology and Immunology, Division of Anatomic Pathology, Washington University in \\ St Louis, School of Medicine, St Louis, MO, USA
}

\begin{abstract}
Sarcomatoid carcinomas are rare malignancies which represent poorly differentiated epithelial tumors that may be difficult to recognize as such. While some cases may have obvious epithelial areas, the sarcomatoid areas are poorly distinguishable from true sarcoma at the light microscopic level and, by immunohistochemistry, often show only limited staining for traditional epithelial markers such as cytokeratin or epithelial membrane antigen. This can be particularly problematic for diagnosis on small biopsy specimens. We sought to assess the diagnostic utility of several immunohistochemical markers of epithelial differentiation including p63, MOC-31, and thyroid transcription factor-1 on sarcomatoid carcinomas of the head and neck (19 cases; 'spindle cell carcinomas'), lung (19 cases), and urinary bladder (11 cases). These results were compared to immunohistochemistry for the traditional epithelial markers pan-cytokeratin and epithelial membrane antigen. Staining for p63 showed the greatest diagnostic utility, positive in 63,50 , and $36 \%$ of head and neck, lung, and urinary bladder sarcomatoid carcinomas, respectively. p63 stains were positive in many cases where immunohistochemistry was negative for both pan-cytokeratin and epithelial membrane antigen. All three alternative markers were quite specific for epithelial differentiation, each staining less than $10 \%$ of the control group of 73 various primary and metastatic sarcomas, melanomas, and benign spindle cell lesions. In conclusion, immunostaining beyond traditional pan-cytokeratin and epithelial membrane antigen may have diagnostic utility in this context.
\end{abstract}

Modern Pathology (2005) 18, 1471-1481. doi:10.1038/modpathol.3800451; published online 10 June 2005

Keywords: p63; MOC-31; TTF-1; sarcomatoid carcinoma; immunohistochemistry

Sarcomatoid carcinomas are rare primary malignancies, which have previously been theorized to either represent 'collision' tumors with epithelial and mesenchymal malignant components developing coexistently, tumors where the epithelial component 'drives' a mesenchymal component, or finally, primary epithelial tumors which have undergone divergent differentiation (metaplasia) as they develop. Accumulated data clearly supports the latter concept, particularly the presence of similar molecular abnormalities in both components and the ability to demonstrate epithelial differentiation in the sarcomatoid component by both electron microscopy and immunohistochemistry. ${ }^{1-5}$ This latter feature has the most practical diagnostic importance because the

Correspondence: Dr JS Lewis Jr, MD, Department of Pathology and Immunology, Washington University in St Louis, $660 \mathrm{~S}$. Euclid Avenue, Box 8118, St Louis, MO, USA.

E-mail: jlewis@path.wustl.edu

Received 3 December 2004; revised 21 April 2005; accepted 22 April 2005; published online 10 June 2005 sarcomatoid areas are poorly distinguishable from true sarcoma at the light microscopic level. However, these tumors often show only focal or no staining for traditional epithelial markers such as pan-cytokeratin or epithelial membrane antigen (EMA) ${ }^{2,4,6-9}$ making definitive diagnosis very difficult. This can be particularly problematic for diagnosis on small biopsy specimens where associated standard carcinoma elements are absent or where growth patterns and tissue involvement cannot be well assessed. Since a number of newer alternative immunohistochemical markers of epithelial differentiation have emerged recently, we sought to investigate their diagnostic utility in this context.

Thyroid transcription factor-1 (TTF-1) is quite specific for tumors of the thyroid gland and lung and is felt to play a role in the differentiation and/or maintenance of bronchiolar and alveolar lining cells. ${ }^{10}$ It has been primarily used for distinguishing lung carcinomas, both primary and metastatic, from other carcinomas. MOC-31 is a membrane glycoprotein isolated from a lung small cell carcinoma cell 
line, which has been shown to stain most normal epithelium and the vast majority of carcinomas. ${ }^{11}$ It has been used primarily in distinguishing adenocarcinomas from mesothelioma in pleural biopsies. ${ }^{12}$

p63 is a transcription factor related to the tumor suppressor gene p53 and has been demonstrated in basal cells of normal glands of the lung and prostate, in normal squamous epithelium, and in normal myoepithelial cells. It is essential for normal development and has an important role in epithelial proliferation and differentiation. ${ }^{13}$ It has been shown to stain the vast majority of squamous cell carcinomas $^{14,15}$ and transitional cell carcinomas ${ }^{16}$ as well as a number of other carcinomas. It is positive in most lung squamous cell carcinomas and in a significant minority of other non-small cell lung carcinomas.

As a result of the difficulty in diagnosis of these tumors and because there is no published data on these newer, alternative markers of epithelial differentiation in such tumors, we sought to assess their diagnostic utility in sarcomatoid carcinomas of the head and neck, lung, and urinary bladder.

\section{Materials and methods}

With institutional review board approval, we compiled 19 cases of sarcomatoid carcinoma ('spindle cell carcinoma') from the head and neck (see Table 1 for demographics), 19 cases from the lung, and 11 cases from the urinary bladder, exclusively from the files of the Division of Anatomic Pathology of Washington University in St Louis, MO from 1990 to 2003 .

The terminology for sarcomatoid carcinomas is confusing and somewhat controversial. We use

Table 1 Demographic information for each head and neck spindle cell carcinoma case

\begin{tabular}{lccl}
\hline Head and neck demographics & Age & Sex & Tumor location \\
\hline 1 & 56 & M & Tonsil \\
2 & 82 & M & Floor of mouth \\
3 & 65 & M & Base of tongue \\
4 & 63 & M & Tonsil \\
5 & 89 & F & Tonsil \\
6 & 68 & M & Gingiva \\
7 & 45 & F & Soft palate \\
8 & 71 & M & Tongue \\
9 & 57 & M & Larynx \\
10 & 78 & F & Gingiva \\
11 & 74 & M & Oropharynx \\
12 & 66 & M & Larynx \\
13 & 60 & M & Pyriform sinus \\
14 & 53 & M & Floor of mouth \\
15 & 69 & F & Tongue \\
16 & 50 & M & Base of tongue \\
17 & 75 & M & Larynx \\
18 & 68 & M & Gingiva \\
19 & 67 & M & Retromolar trigone \\
& & & \\
\hline
\end{tabular}

the term 'sarcomatoid carcinoma' for those tumors occurring at a site where typical carcinomas occur and consisting either partially or entirely of tumor, which by itself is not clearly recognizable as carcinoma at the light microscopic level. Its epithelial nature is discerned by intermingling with a typical carcinoma component (invasive or in situ), by growth patterns, or by demonstration of epithelial differentiation by immunohistochemistry or ultrastructural analysis. These tumors can have spindle cells or pleomorphic cells with multinucleated giant cells and marked atypia and can mimic a number of different lesions such as true sarcoma, spindle cell melanoma, or even benign or reactive spindle cell lesions. We limited this study to those tumors having, either partially or wholly, sarcomatoid features such as spindled or pleomorphic cells (areas without clearly recognizable epithelial differentiation) and excluded those tumors with clearly characterizable and classifiable mesenchymal differentiation (the socalled carcinosarcomas or true malignant mixed tumors). The diagnosis of sarcomatoid carcinoma was based on the above features in each case. It is important to note that in the majority of our cases $(78 \%)$, there was an associated traditional carcinoma component, either in situ or invasive; this being the one criterion establishing these lesions as true carcinomas that is agreed upon by essentially all pathologists. To assess the specificity of immunomarkers in this context, an additional 73 cases of various primary and metastatic sarcomas and melanomas in the lung and at various other sites, granulation tissue polyps of the head and neck, postoperative spindle cell nodules of the bladder, and inflammatory myofibroblastic tumors of the lung and bladder were also analyzed. H\&E slides were reviewed in all cases for verification and documentation of the diagnoses and one block selected for immunostaining. For the carcinomas, $18 / 19(95 \%)$ of the lung, $17 / 19$ (89\%) of the head and neck, and 5/11 (45\%) of the bladder tumors utilized were from definitive resection specimens. For the few biopsy specimens utilized from sarcomatoid carcinomas, all had either a coexistent nonsarcomatoid carcinoma component or had confirmatory immunohistochemistry performed at the time of diagnosis. Of the sarcoma cases utilized, 47/51 (92\%) were from definitive resection specimens. A total of $75 \%(9 / 12)$ of the inflammatory myofibroblastic tumors and all granulation tissue polyps and postoperative spindle cell nodules were from biopsy specimens.

All immunohistochemistry was performed in the pathology research laboratory of the Division of Anatomic Pathology of Washington University in St Louis, MO. All cases were stained for pan-cytokeratin, EMA, p63, and MOC-31. Cases from lung were also stained for TTF-1. Formalinfixed, paraffin embedded blocks were cut into $5-\mu \mathrm{m}$ sections, transferred to poly-lysine-coated 
glass slides, and all cases were stained on a DAKO Automation automated immunostainer utilizing standard autostainer protocols. Antisera were used as follows: pan-cytokeratin 'cocktail' (AE1-AE3; Boehringer Mannheim 1:50 dilution)+CAM 5.2 (Becton Dickinson; 1:50 dilution) + MAK 6 (Triton 1:20 dilution; clones KA4 and UCD/PR-10.11; recognizing cytokeratins $8,14,15,16,18$, and 19); citrate buffer for $8 \mathrm{~min}$ at $\mathrm{pH} 6$ antigen retrieval), EMA (DAKO; citrate buffer for $8 \mathrm{~min}$ at $\mathrm{pH} 6.0$ antigen retrieval), MOC-31 (DAKO; 1:100 dilution; citrate buffer for $8 \mathrm{~min}$ at $\mathrm{pH} 6.0$ and EDTA for $8 \mathrm{~min}$ at $\mathrm{pH} 8.0$ antigen retrieval), TTF-1 (DAKO; 1:400 dilution; clone $8 \mathrm{G} 7 \mathrm{G} 3 / 1$; citrate buffer for $8 \mathrm{~min}$ at $\mathrm{pH} 6.0$ antigen retrieval), and p63 (Neomarkers; 1:100 dilution; clone 4A4; citrate buffer for $8 \mathrm{~min}$ at $\mathrm{pH} 6.0$ and EDTA for $8 \mathrm{~min}$ at $\mathrm{pH} 8.0$ antigen retrieval). The sections were counterstained with hematoxylin. Positive controls consisted of stock tumors known to express the antigen of interest. Appropriate negative controls were also included.

\section{Analysis of Immunostaining}

Slides were analyzed for positive staining and all graded with the following system: $>75 \%$ of cells positive $=4+, \quad 25-75 \%$ positive $=3+, \quad 5-25 \%$ positive $=2+,<5 \%$ positive $=1+$, and no discernible staining $=0$. Sections were viewed by all three authors and graded by the primary author (JSL).

\section{Results}

\section{Head and Neck}

For the 19 sarcomatoid carcinomas (spindle cell carcinomas), all four markers stained the spindle cell component of some of the tumors (see Table 2 and Figures 1 and 2). Staining for p63 was positive in the greatest number of cases $(63 \%)$ followed by staining for EMA (47\%), pan-cytokeratin $(29 \%)$, and MOC-31 (16\%). p63 staining also showed the highest quantitative amount $(2.5+)$ followed by staining for EMA $(1.8+)$, pan-cytokeratin $(1.4+)$, and
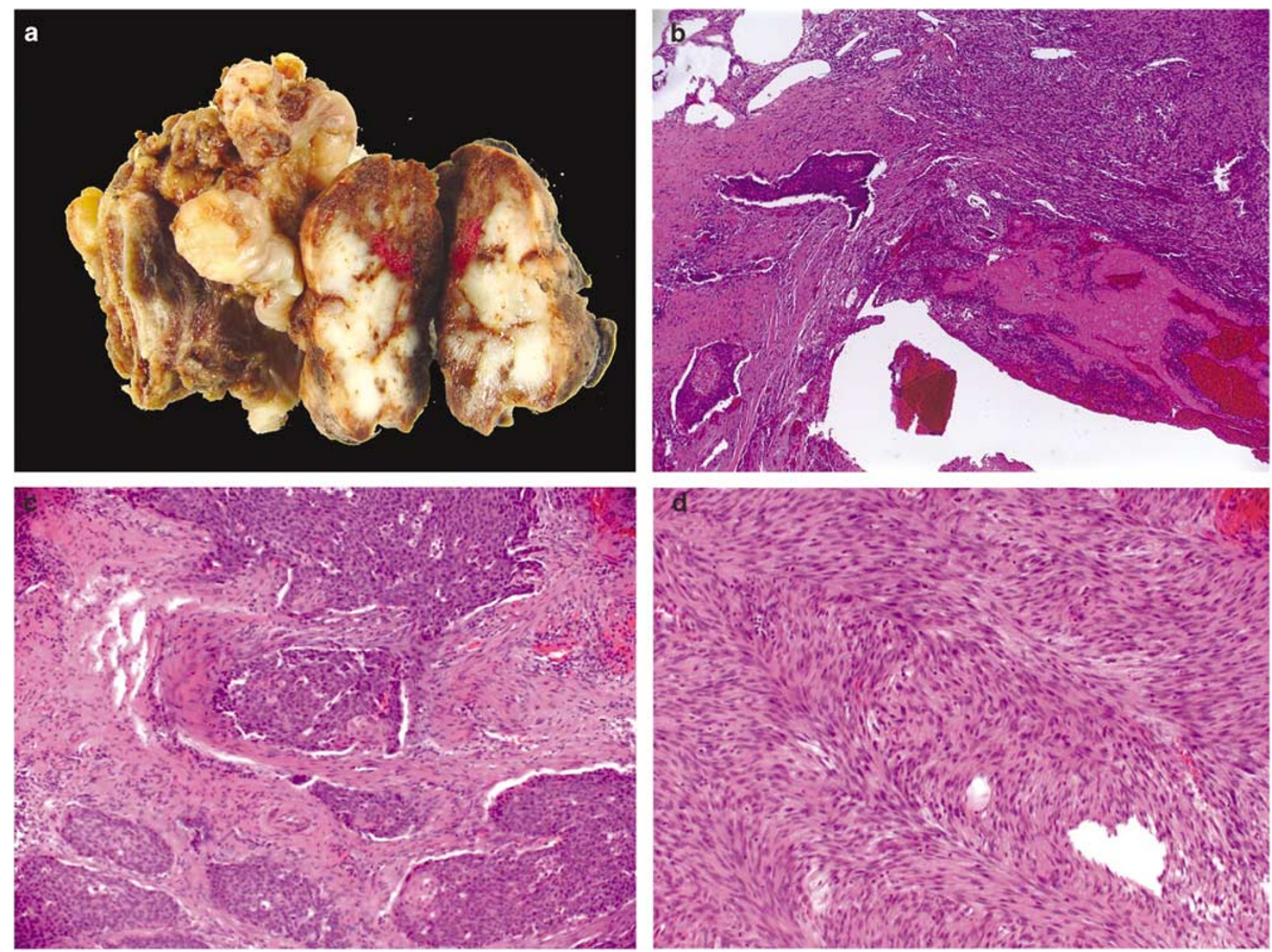

Figure 1 Sarcomatoid (spindle cell) carcinoma of the larynx: (a) Gross photograph showing hemilaryngectomy specimen with polypoid, fleshy, ulcerated tumor (b) Low power showing conventional squamous cell carcinoma component and polypoid spindle cell component (c) High-power conventional squamous cell carcinoma component (d) High-power spindle cell component. (All histology images are hematoxylin and eosin stained; $\mathbf{b}=\times 40$ magnification; $\mathbf{c}=\times 100$ magnification; $\mathbf{d}=\times 200$ magnification). 

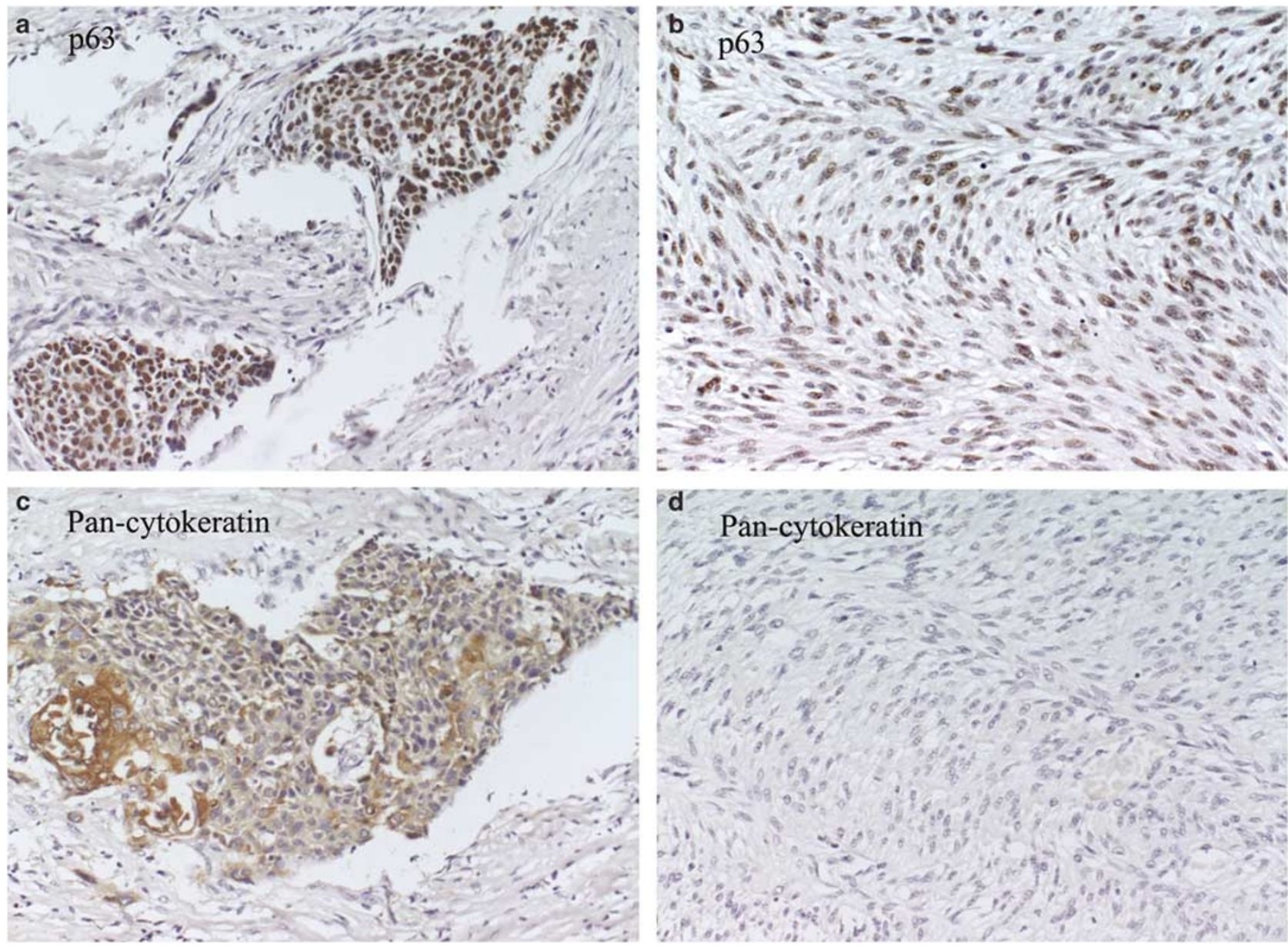

Figure 2 p63 and pan-cytokeratin in Case from Figure 1 showing (a) strong positive p63 staining (nuclear) in the conventional squamous cell carcinoma component, (b) strong positive p63 staining (nuclear) in the spindle cell component, (c) strong positive pan-cytokeratin staining in the conventional squamous cell carcinoma component, (d) total lack of pan-cytokeratin staining in the spindle cell component. (All images $\times 400$ magnification).

MOC-31 $(1+)$. In the six cases where stains for pancytokeratin and EMA were completely negative in the spindled component, staining for p63 was positive in 3/6 (50\%) (Figure 3).

In $14 / 19(74 \%)$ of the cases, a traditional carcinoma component, in situ or invasive, was present in the resection specimen (not always present in the slide chosen for immunostaining). Of these cases, immunostaining of the sarcomatoid component for p63 was positive in 9/14 (64\%), for pan-cytokeratin in $5 / 14(36 \%)$, for EMA in $7 / 14(50 \%)$, and for MOC31 in $3 / 14(21 \%)$. Four cases were negative for all epithelial markers. All of these had a coexistent traditional carcinoma component, in situ or invasive, to confirm their epithelial nature.

The selected slides for staining which had coexistent normal squamous epithelium, squamous cell carcinoma in situ, and traditional invasive squamous cell carcinoma, as expected, showed positive staining in all cases for pan-cytokeratin and EMA, but also were positive in all cases for p63 with
Table 2 Immunohistochemistry results for head and neck spindle cell carcinomas: pan-cytokeratin, EMA, p63, and MOC-31

\begin{tabular}{lcccc}
\hline Head and neck & $\begin{array}{c}\text { Spindle } \\
\text { cell } \\
\text { component }\end{array}$ & $\begin{array}{c}\text { Traditional } \\
\text { squamous } \\
\text { carcinoma } \\
\text { component }\end{array}$ & $\begin{array}{c}\text { Squamous } \\
\text { dysplasial } \\
\text { squamous } \\
\text { carcinoma } \\
\text { in situ }\end{array}$ & $\begin{array}{c}\text { Normal } \\
\text { squamous } \\
\text { epithelium }\end{array}$ \\
\hline Pan-cytokeratin & $5 / 17$ & $7 / 7$ & $6 / 6$ & $8 / 8$ \\
& $29 \%$ & $100 \%$ & $100 \%$ & $100 \%$ \\
& $(1.4+)$ & $(4+)$ & $(4+)$ & $(4+)$ \\
EMA & & & & \\
& $9 / 19$ & $8 / 8$ & $6 / 6$ & $12 / 12$ \\
& $47 \%$ & $100 \%$ & $100 \%$ & $100 \%$ \\
MOC-31 & $(1.8+)$ & $(3.9+)$ & $(3.8+)$ & $(4+)$ \\
& $3 / 19$ & $4 / 7$ & $4 / 6$ & $7 / 12$ \\
& $16 \%$ & $57 \%$ & $67 \%$ & $58 \%$ \\
p63 & $(1.0+)$ & $(2.5+)$ & $(2.8+)$ & $(2.6+)$ \\
& $12 / 19$ & $8 / 8$ & $6 / 6$ & $12 / 12$ \\
& $63 \%$ & $100 \%$ & $100 \%$ & $100 \%$ \\
& $(2.5+)$ & $(4+)$ & $(4+)$ & $(3.9+)$ \\
\hline
\end{tabular}

$\mathrm{EMA}=$ epithelial membrane antigen. 

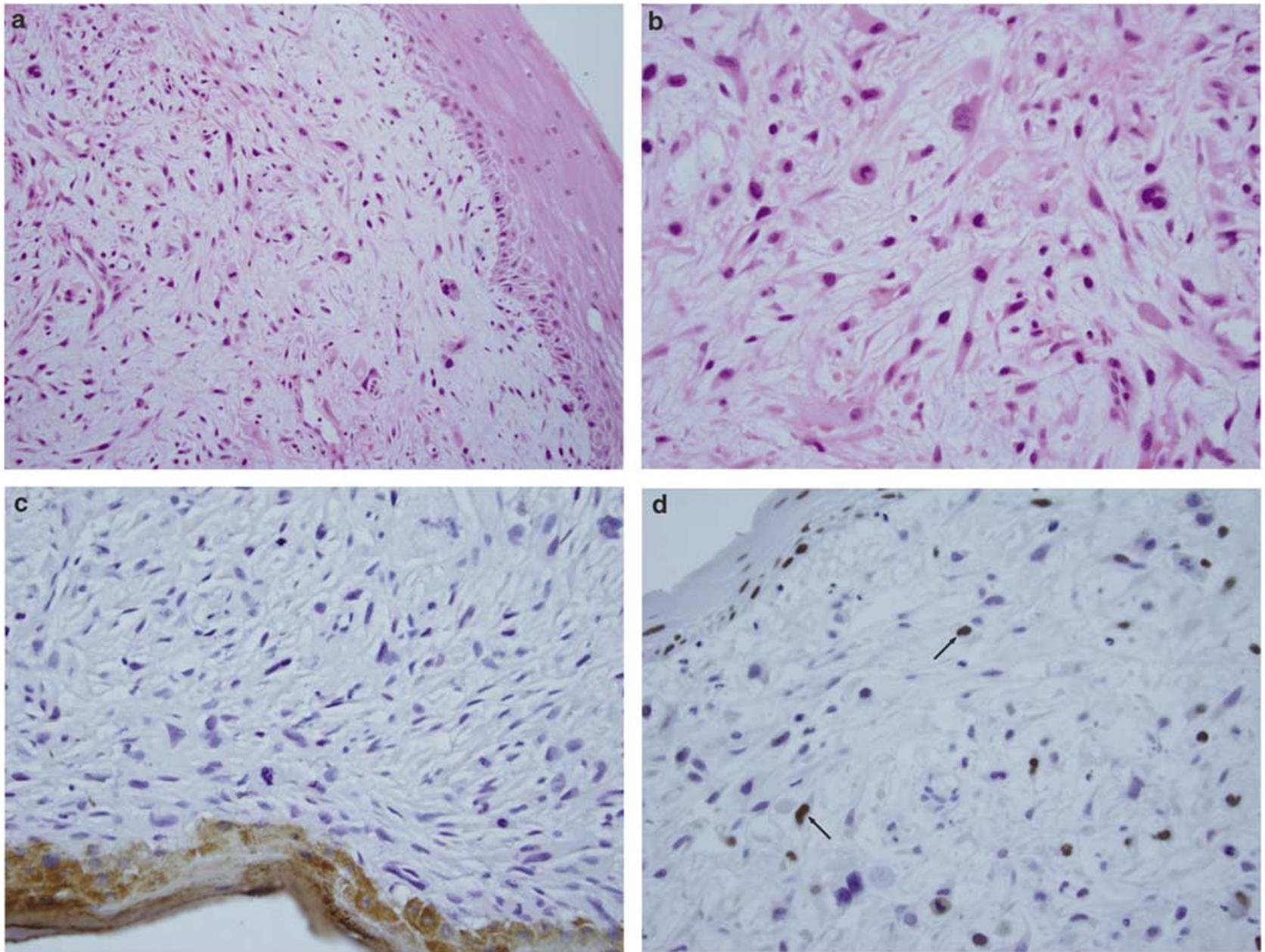

Figure 3 p63 and pan-cytokeratin in additional highly pleomorphic spindle cell carcinoma: (a) and (b) Low and high power showing benign surface squamous epithelium with underlying pleomorphic and spindle cell malignancy, (c) negative pan-cytokeratin immunohistochemistry, and (d) p63 immunohistochemistry showing frequent strongly positive pleomorphic tumor cells. (a= $\times 200$ magnification; hematoxylin and eosin stained; $\mathbf{b}=\times 400$ magnification; hematoxylin and eosin stained; $\mathbf{c}$ and $\mathbf{d}=\times 400$ magnification).

staining of the basal layers of normal squamous epithelium and diffuse staining of squamous cell carcinoma in situ and invasive squamous cell carcinoma. Staining for MOC-31 was much less consistent with only $54 \%$ of normal squamous epithelium, also of only the basal portions, and 57 and $66 \%$ of squamous cell carcinoma in situ and invasive squamous cell carcinoma being positive.

\section{Lung}

In all 19 cases, immunostains for each marker were positive in the sarcomatoid components of some of the examined tumors. Staining for pan-cytokeratin was positive in the greatest number of cases $(79 \%)$ followed by staining for EMA (68\%), p63 (50\%), MOC-31 (42\%), and TTF-1 (26\%) (see Table 3). Staining for EMA showed the highest quantitative amount $(2.9+)$ followed by staining for p63 $(2.5+)$, pan-cytokeratin $(2.1+)$, and TTF-1 and MOC-31 $(1.8+$ each). In the four cases where staining for pan-cytokeratin and EMA were both completely negative in the spindled component, staining for p63 was positive in two $(50 \%)$, MOC-31 in one $(25 \%)$, and TTF-1 in zero.

In $15 / 19(79 \%)$ of the cases, a traditional carcinoma component was present in the resection specimen (not always present in the slide chosen for immunostaining). Of these cases, immunostaining of the sarcomatoid component for p63 was positive in $7 / 15$ (47\%), for pan-cytokeratin in 13/15 (87\%), for EMA in 12/15 (80\%), for MOC-31 in 7/15 $(47 \%)$, and for TTF-1 in 5/15 (33\%). Two cases were negative for all epithelial markers. One of these had a coexistent traditional invasive carcinoma component and the other did not. This latter case was diagnosed on the basis of growth pattern and cytologic features. 
Table 3 Immunohistochemistry results for lung sarcomatoid carcinomas: Pan-cytokeratin, EMA, p63, TTF-1, and MOC-31

\begin{tabular}{|c|c|c|c|c|c|}
\hline Lung & Pan-cytokeratin & $E M A$ & $T T F-1$ & $M O C-31$ & p63 \\
\hline $\begin{array}{l}\text { Sarcomatoid } \\
\text { carcinoma (nonsarcomatoid component) }\end{array}$ & $\begin{array}{l}10 / 10 \\
100 \% \\
(3.2+)\end{array}$ & $\begin{array}{l}10 / 10 \\
100 \% \\
(3.1+)\end{array}$ & $\begin{array}{c}7 / 10 \\
70 \% \\
(2.4+)\end{array}$ & $\begin{array}{l}10 / 10 \\
100 \% \\
(3.3+)\end{array}$ & $\begin{array}{c}5 / 10 \\
50 \% \\
(2.8+)\end{array}$ \\
\hline Sarcomatoid carcinoma (sarcomatoid component) & $\begin{array}{c}15 / 19 \\
79 \% \\
(2.1+)\end{array}$ & $\begin{array}{c}13 / 19 \\
68 \% \\
(2.9+)\end{array}$ & $\begin{array}{c}5 / 19 \\
26 \% \\
(1.8+)\end{array}$ & $\begin{array}{c}8 / 19 \\
42 \% \\
(1.8+)\end{array}$ & $\begin{array}{c}10 / 20 \\
50 \% \\
(2.5+)\end{array}$ \\
\hline
\end{tabular}

EMA = epithelial membrane antigen; TTF-1 = thyroid transcription factor-1.

The conventional carcinoma component was present on the stained section in only 10 cases as follows: adenocarcinoma (two cases), squamous cell carcinoma (two cases), adenosquamous carcinoma (two cases), large cell undifferentiated carcinoma (three cases), and mixed adenocarcinoma/neuroendocrine carcinoma (one case). This conventional carcinoma was immunopositive for pan-cytokeratin, EMA, and MOC-31 in all cases. Seven out of 10 were immunopositive for TTF-1 and 5/10 for p63.

Staining for pan-cytokeratin, EMA, MOC-31, TTF1 , and p63 was positive in the normal bronchiolar epithelium of all cases where it was present and staining for MOC-31 was positive in normal pneumocytes on a relatively consistent basis.

\section{Urinary Bladder}

For these cases, only three of the four markers stained the sarcomatoid components of some of the examined tumors. Staining for pan-cytokeratin was positive in the most cases $(80 \%)$ followed by staining for EMA (73\%), and p63 (36\%). Staining for MOC-31 was negative in all of the tumors (see Table 4). Staining for p63 showed the highest quantitative amount $(2.8+)$ followed by staining for pan-cytokeratin $(2.6+)$, and EMA $(2.5+)$. In the two cases where staining for pan-cytokeratin and EMA were both completely negative in the spindled component, staining for p63 was positive in one case.

In nine of the 11 cases, there was an associated traditional carcinoma component present in the resection specimen (not always present in the slide chosen for immunostaining), either in situ or invasive. Of these nine, immunostaining in the sarcomatoid component for p63 was positive in $3 / 9(33 \%)$, for pan-cytokeratin in $7 / 9(78 \%)$, for EMA in 6/9 (66\%), and for MOC-31 in 0/9 (0\%). All markers were strongly and diffusely immunopositive in residual normal transitional epithelium when present, as well. One case was negative for all epithelial markers. This had a coexistent traditional carcinoma component, both in situ and invasive.
Table 4 Immunohistochemistry results for urinary bladder sarcomatoid carcinomas: pan-cytokeratin, EMA, p63, and MOC-31

\begin{tabular}{lcccc}
\hline Urinary bladder & $\begin{array}{c}\text { Spindle } \\
\text { cell } \\
\text { component }\end{array}$ & $\begin{array}{c}\text { Traditional } \\
\text { carcinoma } \\
\text { component }\end{array}$ & $\begin{array}{c}\text { Flat } \\
\text { carcinoma } \\
\text { in situ }\end{array}$ & $\begin{array}{c}\text { Normal } \\
\text { urothelium }\end{array}$ \\
\hline Pan-cytokeratin & $8 / 10$ & 0 & $1 / 1$ & $8 / 8$ \\
& $80 \%$ & & $100 \%$ & $100 \%$ \\
& $(2.6+)$ & & $(4+)$ & $(4+)$ \\
EMA & $8 / 11$ & 0 & $1 / 1$ & $8 / 8$ \\
& $73 \%$ & & $100 \%$ & $100 \%$ \\
& $(2.5+)$ & & $(4+)$ & $(4+)$ \\
MOC-31 & $0 / 11$ & 0 & $0 / 1$ & $8 / 8$ \\
& $0 \%$ & & $0 \%$ & $100 \%$ \\
& & & & $(3.8+)$ \\
p63 & $4 / 11$ & 0 & $1 / 1$ & $7 / 7$ \\
& $36 \%$ & & $100 \%$ & $100 \%$ \\
& $(2.8+)$ & & $(4+)$ & $(4+)$ \\
\hline
\end{tabular}

EMA $=$ epithelial membrane antigen.

\section{Control Cases}

For the lung, staining was performed for all five markers on 22 primary and metastatic tumors (18 sarcomas and four melanomas). Otherwise, staining was performed for the four main markers (excluding TTF-1) on an additional 27 control cases (23 sarcomas and four melanomas) from various sites including soft tissue, head and neck, and visceral sites (see Tables 5 and 6). Staining for EMA showed the least epithelial specificity of all of the markers, positive in 6/18 sarcomas in the lung and 13/23 of the other sarcomas, including $5 / 7$ synovial sarcomas, 7/17 leiomyosarcomas, 3/8 malignant fibrous histiocytomas, and 2/5 malignant peripheral nerve sheath tumors. Staining for pan-cytokeratin was also positive in various sarcomas including $4 / 7$ synovial sarcomas and 2/17 leiomyosarcomas. The alternative epithelial markers were more specific. Staining for p63 was positive in only one synovial sarcoma, one malignant peripheral nerve sheath tumor, and one malignant fibrous histiocytoma. None of the 17 leiomyosarcomas were immunopositive including 
Table 5 Immunohistochemistry results for sarcoma, melanoma, and benign spindle cell lesions: pan-cytokeratin, EMA, p63, TTF-1, and MOC-31

\begin{tabular}{|c|c|c|c|c|c|}
\hline Control cases & Various sarcomas & $\begin{array}{l}\text { Lung sarcomas } \\
\text { (primary and } \\
\text { metastatic) }\end{array}$ & $\begin{array}{l}\text { Melanomas } \\
\text { (primary and } \\
\text { metastatic) }\end{array}$ & $\begin{array}{c}\text { Benign spindle cell } \\
\text { lesions }^{\mathrm{a}}\end{array}$ & Total \\
\hline Pan-cytokeratin & $\begin{array}{l}3 / 23 \\
13 \% \\
(2.7+)\end{array}$ & $\begin{array}{l}3 / 18 \\
17 \% \\
(2.6+)\end{array}$ & $\begin{array}{l}0 / 8 \\
0 \%\end{array}$ & $\begin{array}{l}2 / 20 \\
10 \%\end{array}$ & $\begin{array}{l}8 / 69 \\
12 \%\end{array}$ \\
\hline EMA & $\begin{array}{l}13 / 23 \\
57 \% \\
(2.3+)\end{array}$ & $\begin{array}{c}6 / 18 \\
33 \% \\
(2.3+)\end{array}$ & $\begin{array}{l}0 / 8 \\
0 \%\end{array}$ & $\begin{array}{l}3 / 21 \\
10 \%\end{array}$ & $\begin{array}{c}22 / 70 \\
31 \%\end{array}$ \\
\hline MOC-31 & $\begin{array}{c}4 / 25 \\
16 \% \\
(1.3+)\end{array}$ & $\begin{array}{c}1 / 18 \\
6 \% \\
(2+)\end{array}$ & $\begin{array}{c}1 / 8 \\
13 \%\end{array}$ & $\begin{array}{l}0 / 21 \\
0 \%\end{array}$ & $\begin{array}{l}6 / 72 \\
8 \%\end{array}$ \\
\hline p63 & $\begin{array}{l}2 / 23 \\
9 \% \\
(1+)\end{array}$ & $\begin{array}{l}1 / 18 \\
6 \% \\
(4+)\end{array}$ & $\begin{array}{l}0 / 8 \\
0 \%\end{array}$ & $\begin{array}{l}1 / 22 \\
5 \%\end{array}$ & $\begin{array}{l}4 / 71 \\
6 \%\end{array}$ \\
\hline TTF-1 & NP & $\begin{array}{l}1 / 18 \\
6 \% \\
(4+)\end{array}$ & $\begin{array}{l}0 / 4 \\
0 \%\end{array}$ & $\begin{array}{l}0 / 9^{b} \\
0 \%\end{array}$ & $\begin{array}{l}1 / 31 \\
3 \%\end{array}$ \\
\hline
\end{tabular}

$\mathrm{NP}=$ not performed; EMA = epithelial membrane antigen; TTF-1 = thyroid transcription factor-1.

${ }^{\mathrm{a}}$ Mixture of granulation tissue polyps of the head and neck, postoperative spindle cell lesions of the urinary bladder, and inflammatory myofibroblastic tumors of the bladder and lung.

${ }^{\mathrm{b}}$ All lung inflammatory myofibroblastic tumors.

Table 6 Immunohistochemistry results for specific sarcoma types: pan-cytokeratin, EMA, p63, TTF-1, and MOC-31

\begin{tabular}{|c|c|c|c|c|}
\hline $\begin{array}{l}\text { Specific Control } \\
\text { Cases }\end{array}$ & Leiomyosarcoma & Synovial sarcoma & $\begin{array}{l}\text { Malignant fibrous } \\
\text { histiocytoma }\end{array}$ & $\begin{array}{c}\text { Malignant } \\
\text { peripheral nerve } \\
\text { sheath tumor }\end{array}$ \\
\hline Pan-cytokeratin & $\begin{array}{l}2 / 17 \\
12 \%\end{array}$ & $\begin{array}{c}4 / 7 \\
57 \%\end{array}$ & $\begin{array}{l}0 / 8 \\
0 \%\end{array}$ & $\begin{array}{l}0 / 5 \\
0 \%\end{array}$ \\
\hline EMA & $\begin{array}{l}7 / 17 \\
41 \%\end{array}$ & $\begin{array}{c}5 / 7 \\
71 \%\end{array}$ & $\begin{array}{c}3 / 8 \\
38 \%\end{array}$ & $\begin{array}{c}2 / 5 \\
40 \%\end{array}$ \\
\hline MOC-31 & $\begin{array}{l}1 / 17 \\
6 \%\end{array}$ & $\begin{array}{c}1 / 7 \\
14 \%\end{array}$ & $\begin{array}{c}1 / 8 \\
13 \%\end{array}$ & $\begin{array}{c}2 / 5 \\
40 \%\end{array}$ \\
\hline p63 & $\begin{array}{l}0 / 17 \\
0 \%\end{array}$ & $\begin{array}{c}1 / 7 \\
14 \%\end{array}$ & $\begin{array}{c}1 / 8 \\
13 \%\end{array}$ & $\begin{array}{c}1 / 5 \\
20 \%\end{array}$ \\
\hline TTF-1 & $\begin{array}{c}0 / 10 \\
0 \%\end{array}$ & $\begin{array}{c}1 / 5 \\
20 \%\end{array}$ & NP & NP \\
\hline
\end{tabular}

$\mathrm{NP}=$ not performed; EMA = epithelial membrane antigen; TTF-1 = thyroid transcription factor-1.

two from the urinary bladder. Staining for MOC-31 was positive in one leiomyosarcoma, one synovial sarcoma, one malignant fibrous histiocytoma, and two malignant peripheral nerve sheath tumors. Finally, staining for TTF-1 was positive in only one case, a synovial sarcoma. Of note, all synovial sarcomas which were immunopositive for the alternative epithelial markers showed a distinctly epithelioid H\&E component. Except for a single case with focal $(1+)$ staining for MOC-31, the eight melanomas, four of which were primary lesions in the head and neck, were negative for epithelial markers.

Benign mimics of spindle cell carcinoma were also immunostained (see Table 5), including eight cases of head and neck granulation tissue polyps. All of these were negative for the epithelial immunohistochemistry. An additional five cases of postoperative spindle cell lesions/inflammatory myofibroblastic tumors of the bladder were immu- 
nostained and all were also negative for the markers. Finally, nine cases of inflammatory myofibroblastic tumor of the lung were immunostained and only one case showed focal, weak p63 staining of the spindle cells. These tumors also showed, in a few cases, focal pan-cytokeratin and EMA staining of the spindle cells as has been previously observed. No convincing inflammatory myofibroblastic tumors of the upper aerodigestive tract/head and neck region were found in the case files to analyze.

\section{Discussion}

Sarcomatoid carcinomas are uncommon and often diagnostically challenging tumors that can arise at almost any site where typical carcinomas arise but which are more commonly seen in the head and neck region, lung, and urinary bladder. The presence of spindled or pleomorphic cells without obvious epithelial differentiation by light microscopy in most cases makes these diagnostically difficult to discern from primary spindle cell sarcomas, melanomas, or even reactive spindle cell lesions, which occur at the same sites. Growth patterns and the presence of a traditional invasive (nonsarcomatoid) or in situ carcinoma component are very important for the diagnosis. In addition, immunohistochemistry for markers of epithelial differentiation may be helpful, and sometimes are critical, for the diagnosis, particularly on small biopsy specimens. Immunostaining for pan-cytokeratin and EMA may be very useful but are not uniformly positive in these tumors. Also, EMA is not completely specific, with varying amounts of staining in true sarcomas, ${ }^{17-21}$ leaving cases where other diagnostic tools might be helpful. There is also well-documented expression of mesenchymal markers such as vimentin, smooth muscle actin, and desmin in sarcomatoid carcinomas. ${ }^{6,2,9}$ It is difficult to be definitive about the diagnosis of carcinoma in a spindle cell lesion where the pathologist cannot demonstrate any epithelial phenotype even though the clinical setting may strongly favor that diagnosis (see below), so newer and more sensitive markers of epithelial differentiation may be useful in these tumors.

We found p63 staining to be the most diagnostically useful, particularly in head and neck sarcomatoid carcinomas (spindle cell carcinomas) where it showed the highest sensitivity $(63 \%)$ of all of the markers and was positive in several of the cases where immunostaining for pan-cytokeratin and EMA were completely negative. When positive, p63 also showed stronger, more diffuse staining than pan-cytokeratin or EMA. Some other recent data is available for p63 in spindle cell carcinomas of the head and neck, ${ }^{22}$ which is comparable to our own. Krassilnik et $a l^{22}$ demonstrated positive immunostaining in 10/19 (53\%) cases of head and neck spindle cell carcinoma and also showed stronger quantitative staining than the cytokeratins. They did not address any issues of specificity, however. In addition, the nuclear staining is easier to interpret than cytoplasmic when it is focal because spurious staining almost never occurs in a nuclear localized pattern.

The utility of p63 immunostaining in the lung is slightly less, marking $50 \%$ of these tumors, but also being positive in a few cases where immunostaining for pan-cytokeratin and EMA was completely negative. In the urinary bladder, staining for p63 was positive in only $36 \%$ of cases but was positive in one case where stains for pan-cytokeratin and EMA were completely negative.

TTF-1, although it was reactive in a small but significant percentage of the sarcomatoid carcinomas, was generally focal and was negative in all cases where staining for pan-cytokeratin and EMA were negative. This may well reflect the fact that many of the tumors which had a sarcomatoid carcinoma component otherwise had squamous differentiation, which usually lacks TTF-1 expression.

Immunostaining for MOC-31, despite being positive in most epithelium in the human body and most carcinomas, also did not perform particularly well compared to pan-cytokeratin and EMA, being positive in only $16 \%$ of head and neck sarcomatoid carcinomas, none of the urinary bladder cases, and only a fraction of the lung sarcomatoid carcinomas. It too, like TTF-1, is more consistently immunopositive in glandular-type epithelium, and its lack of staining may be a reflection of the fact that many of these tumors arose in the setting of squamous cell carcinoma, especially in the head and neck.

The definition of our cases of sarcomatoid carcinoma included a few cases where no traditional carcinoma component was present on any specimen. These cases were diagnosed using pan-cytokeratin and/or EMA immunostaining at the time to confirm them as carcinomas. However, the vast majority (38/49 or $78 \%$ ) of our cases did have some form of traditional carcinoma, either invasive or in situ, associated with the sarcomatoid carcinoma, the presence of which would have to be considered the 'gold standard' for this diagnosis. The data were comparable in these groups to the overall data.

Of additional importance, in what is the only data of its kind thus far, all of the alternative epithelial markers showed specificity profiles at least as good as pan-cytokeratin and EMA, and better in most cases, particularly compared to EMA. Only very few sarcomas were positive for p63, MOC-31, or TTF-1. Except for synovial sarcomas with obvious epithelial differentiation on $\mathrm{H} \& \mathrm{E}$, all cases showed focal staining $(1$ or $2+)$. Immunostaining for p63, in particular, was positive in only $3 / 49$ sarcomas and negative in all eight melanomas. Overall, staining for p63 had a specificity of 94\%, pan-cytokeratin of $88 \%$, EMA of $65 \%$, MOC-31 of $92 \%$, and TTF-1 of $97 \%$. Although the numbers are still relatively small, our data suggest that immunostaining for 
p63 is a very specific marker for epithelial differentiation, making it very useful in the diagnosis of sarcomatoid carcinomas.

We acknowledge that in some cases of sarcomatoid carcinoma, an extended panel of keratin immunostaining can show positivity where antibodies to pan-cytokeratin (AE1/AE3, CAM 5.2, and MAK-6) typically used in the clinical practice setting, may be negative. We simply wanted to compare the alternative markers to what is typically used in a practical sense for the diagnostic surgical pathologist. Most institutions do not provide immunostaining for an exhaustive panel of cytokeratins, nor do pathologists in routine practice utilize such extended panels. Also, one would worry about doing a high number of immunostains, a situation where one is likely to get a spurious result with one or more in a lesion that is normally not pan-cytokeratin positive, and then relying on that as a diagnostic feature, could result in erroneous diagnoses of lesions that are not sarcomatoid carcinomas.

There are rare cases of mucosal-based malignant spindle cell lesions in the head and neck or of the lung or bladder where there is no traditional component of in situ or invasive carcinoma and where all immunohistochemistry for epithelial markers is negative. This was the particular scenario this paper was meant to address. In such cases, if we can diagnose another malignant spindle cell lesion, we classify the tumor as such. Particularly, melanoma should be ruled out or in and is usually not problematic. Beyond this, in all three sites, we would sign these out as 'malignant spindle cell neoplasm' and favor one diagnosis or another based on impression and/or clinical data or other information. This happens most often on biopsy, and subsequent resection often provides more information and the necessary morphology to make a definitive diagnosis. In the head and neck region, given the rarity of mucosal-based spindle cell sarcomas, spindle cell carcinoma is almost always the favored diagnosis.

Rare benign lesions can mimic spindle cell carcinoma, including granulation tissue polyps of the head and neck. These sometimes have spindled stromal and endothelial cells and with their polypoid and ulcerated conformation, can closely resemble blander-appearing spindle cell carcinomas. Another potential mimic is the inflammatory myofibroblastic tumor, which can occur at any site. These typically consist of a proliferation of fibroblasts and myofibroblasts with an associated, and usually prominent, inflammatory cell component including plasma cells, lymphocytes, neutrophils, eosinophils, and histiocytes. The spindle cells usually have oval nuclei with vesicular chromatin and prominent nucleoli but do not demonstrate marked nuclear atypia. Although they can mimic spindle cell carcinoma, the presence of plasma cells or eosinophils would be quite unusual and the lack of cytologic atypia in inflammatory myofibroblastic tumors is also a clue to discern them from spindle cell carcinoma. The clinical setting is also often quite different. For example, often these are younger patients without a smoking history. Immunohistochemistry typically shows positive staining for vimentin and smooth muscle actin and, although usually negative, immunohistochemistry can be positive, including for cytokeratin in a significant proportion of cases ${ }^{23}$ but, in our experience, the blander-appearing spindle cell carcinomas, will often demonstrate more consistent and strong epithelial marker staining. Finally, many inflammatory myofibroblastic tumors are positive for the ALK-1 protein by immunohistochemistry due to abnormalities of chromosome $2 \mathrm{p} 23 .{ }^{23,24}$ Spindle cell carcinomas are negative for ALK-1 in the few cases examined. ${ }^{24,25}$ We found only one of $12(1 / 12)$ cases to be very focally positive for p63 and none for TTF-1 and MOC-31.

Our recommendation based on our findings would be to utilize p63 as an additional marker of epithelial differentiation in cases where immunohistochemistry is indicated for the diagnosis of a spindle cell neoplasm where sarcomatoid carcinoma is in the differential diagnosis. The utility is associated with positive staining, as with other epithelial markers. It can be particularly useful in cases where leiomyosarcoma is in the differential diagnosis because it is well known that these lesions can express EMA (7/17 or $41 \%$ in our series) or pancytokeratin $(2 / 17$ or $12 \%$ in our study) focally and sarcomatoid carcinomas have been shown to express smooth muscle markers in a certain percentage of cases. Immunostaining for p63 was negative in all 17 cases of leiomyosarcoma in our series. Immunostaining for p63 can either be ordered up front along with pan-cytokeratin and EMA, or ordered as a second line in cases where both of these markers come back negative. Immunostains for TTF-1 and MOC-31, as we have shown, are also quite specific for epithelial differentiation, and when positive in sarcomatoid carcinoma, are helpful, so may be utilized in certain clinical situations as well, particularly in the lung.

It is interesting to speculate on the differentiation of sarcomatoid carcinomas, particularly with respect to their loss or retention of epithelial characteristics. Accumulated data clearly points to the concept that these are truly carcinomas, which have lost much of their epithelial characteristics (or 'dedifferentiated' if one prefers that terminology). Since what we are primarily evaluating by light microscopy is an expression of structural molecules, proteins, etc, then the lack of these structural features under the microscope would suggest a loss of expression of structural molecules such as pan-cytokeratin, which are intermediate filaments, or EMA and MOC-31, which, although their exact functions are unknown, reside in the plasma membrane with presumably structural or functional roles. One could speculate 
that transcription factors, like p63 and TTF-1, might be more likely to be retained in a tumor that is poorly differentiated since they are likely to regulate many more processes in cell differentiation and gene expression and do not have a direct role in determining the structure and morphology of tumor cells. From our results, particularly in the head and neck, this appears to be the case.

Sarcomatoid carcinomas will continue to be diagnostically challenging for the surgical pathologist. With expression profiling and the identification of newer markers of epithelial differentiation in the future, other immunohistochemical markers might be more diagnostically useful in their differential diagnosis. For now, we must continue to rely on identifying typical growth patterns in these tumors, conventional carcinoma components or associated dysplasia/in situ carcinoma, and, in challenging cases, utilize immunohistochemistry. Immunostaining for p63 is another useful marker in addition to pan-cytokeratin and EMA for this purpose, particularly in the head and neck, but also potentially in select cases in the lung and urinary bladder.

\section{Acknowledgement}

We would like to extend a special thanks to Prosperidad Amargo for her excellent work with the immunohistochemistry for this study.

\section{References}

1 Choi HR, Sturgis EM, Rosenthal DI, et al. Sarcomatoid carcinoma of the head and neck; molecular evidence for evolution and progression from conventional squamous cell carcinoma. Am J Surg Pathol 2003;27: 1216-1220.

2 Nappi O, Glasner SD, Swanson PE, et al. Biphasic and monophasic sarcomatoid carcinomas of the lung. A reappraisal of 'carcinosarcomas' and 'spindle cell carcinomas'. Am J Clin Pathol 1994;102: 331-340.

3 Nappi O, Wick MR. Sarcomatoid neoplasms of the respiratory tract. Semin Diagn Pathol 1993;10: 137-147.

4 Torenbeek R, Blomjous CEM, deBruin PC, et al. Sarcomatoid carcinoma of the urinary bladder-clinicopathologic analysis of 18 cases with immunohistochemical and electron microscopic findings. Am J Surg Pathol 1994;18:241-249.

5 Torenbeek R, Hermsen MAJA, Meijer GA, et al. Analysis by comparative hybridization of epithelial and spindle cell components in sarcomatoid carcinoma and carcinosarcoma: histogenetic aspects. J Pathol 1999;189:338-343.

6 Ikegami H, Iwasaki H, Ohjimi Y, et al. Sarcomatoid carcinoma of the urinary bladder: a clinicopathologic and immunohistochemical analysis of 14 patients. Hum Pathol 2000;31:332-340.
7 Jones EC, Young RH. Myxoid and sclerosing sarcomatoid transitional cell carcinoma of the urinary bladder: a clinicopathologic and immunohistochemical study of 25 cases. Mod Pathol 1997;10: 908-916.

8 Matsui K, Kitagawa M, Miwa A. Lung carcinoma with spindle cell components; sixteen cases examined by immunohistochemistry. Hum Pathol 1992;23: 1289-1297.

9 Thompson LDR, Weineke JA, Miettinen M, et al. Spindle cell (sarcomatoid) carcinomas of the larynx; a clinicopathologic study of 187 cases. Am J Surg Pathol 2002;26:153-170.

10 Nakamura N, Miyagi E, Murata S, et al. Expression of thyroid transcription factor 1 in normal and neoplastic lung tissue. Mod Pathol 2002;15:1058-1067.

11 Souhami RL, Beverley PC, Bobrow LG, et al. Antigens of lung cancer: results of the second international workshop on lung cancer antigens. J Natl Cancer Inst 1991;83:609-612.

12 Edwards C, Oates J. OV632 and MOC 31 in the diagnosis of mesothelioma and adenocarcinoma: an assessment of their use in formalin fixed and paraffin wax embedded material. J Clin Pathol 1995;48: 626-630.

13 DiComo CJ, Urist MJ, Babayan I, et al. p63 expression profiles in human normal and tumor tissues. Clin Cancer Res 2002;8:494-501.

14 Pruneri G, Pignataro L, Manzotti M, et al. p63 in laryngeal squamous cell carcinoma: evidence for a role in TA-p63 down regulation in tumorigenesis and lack of prognostic implications of p63 immunoreactivity. Lab Invest 2002;82:1327-1334.

15 Wang BY, Gil J, Kaufman D, et al. p63 in pulmonary epithelium, pulmonary squamous neoplasms, and other pulmonary tumors. Hum Pathol 2002;33: 921-926.

16 Langner C, Ratschek M, Tsybrovskyy O, et al. p63 immunoreactivity distinguishes upper urinary tract transitional-cell carcinoma and renal-cell carcinoma even in poorly differentiated tumors. J Histochem Cytochem 2003;51:1097-1099.

17 Deyrup AT, Montgomery E, Fisher C. Leiomyosarcoma of the kidney: a clinicopathologic study. Am J Surg Pathol 2004;28:178-182.

18 Meis JM, Enzinger FM, Martz KL, et al. Malignant peripheral nerve sheath tumors (malignant schwannomas) in children. Am J Surg Pathol 1992;16:694-707.

19 Moran CA, Suster A, Abbondanzo SL, et al. Primary leiomyosarcomas of the lung: a clinicopathologic and immunohistochemical study of 18 cases. Mod Pathol 1997;10:121-128.

20 Rosenberg AE, O’Connell JX, Dickersin GR, et al. Expression of epithelial markers in malignant fibrous histiocytoma of the musculoskeletal system: an immunohistochemical and electron microscopic study. Hum Pathol 1993;24:284-293.

21 Rubin BP, Fletcher CD. Myxoid leiomyosarcoma of soft tissue, an underrecognized variant. Am J Surg Pathol 2000;24:927-936.

22 Krassilnik N, Gologan O, Ghali V, et al. p63 and p16 expression in spindle cell carcinomas of the head and neck. Mod Pathol 2004;17(Suppl 1):226A (Abstract \#953).

23 Coffin CM, Watterson J, Priest JR, et al. Extrapulmonary inflammatory myofibroblastic tumor (inflammatory pseudotumor). A clinicopathologic and immunohisto- 
chemical study of 84 cases. Am J Surg Pathol 1995;20: 859-872.

24 Cook JR, Dehner LP, Collins MH, et al. Anaplastic lymphoma kinase (ALK) expression in the inflammatory myofibroblastic tumor: a comparative immunohistochemical study. Am J Surg Pathol 2001;25:1364-1371.
25 Freeman A, Geddes N, Munson P, et al. Anaplastic lymphoma kinase (ALK1) staining and molecular analysis in inflammatory myofibroblastic tumours of the bladder: a preliminary clinicopathological study of nine cases and review of the literature. Mod Pathol 2004;17:765-771. 